\title{
EFFECT OF PRELOADING ON LOWER JAW IMPLANT
}

János Simonovics ${ }^{1}$, Péter Bujtár ${ }^{2}$, Károly Váradi ${ }^{1}$

${ }^{1}$ Department of Machine and Product Design, Budapest University of Technology and Economics

${ }^{2}$ Department of Maxillofacial Surgery, University Hospitals of Leicester

janos@simonovics.bu

\begin{abstract}
The procedure of mandible resection is basically unavoidable in case of cancer in the field of oral surgery. The reinforcement and the reconstruction of the jaw closest to its original condition is very challenging. Considering the load properties the examination of the plates used in the reconstruction is highly important. The cadaver examination procedure however is very difficult to execute, therefore the use of the Finite Element Analysis proves rather supportive. Fast recovery can be achieved by applying the implants correctly. Furthermore brakeage resulting from high loads in the plates can be avoided. The goal is to examine and understand the Nonlocking screwing technique used with plate implants in different mandible resections and reconstructions, focusing on the preloaded force. Furthermore the study and comparison of different stress that arise in different cases.
\end{abstract}

A toothless mandible was used for the creation of the Finite Element Analysis model. The data was provided for the model by CBCT. During the creation of the model we separated four different resection areas to which we used the plates with the Non-Locking screwing technique, also used in clinical reconstructions. For the preload of the plates we used different preloaded forces on the screws. We considered and used boundary conditions complying with the anatomical structure. The muscles required for chewing were transferred to the model and used as main loads. The bone structure is heterogenic and the bone density is based on CBCT.

The preloaded forces have a major role in the stresses arising in the bone during the use of Nonlocking technique. The comparison of the resection techniques can provide valuable information regarding the preloaded condition and the following chewing load condition connected to the main loads in the bone.

Those measurements that are important from biomechanical point of view and would prove difficult or impossible with in vivo or in vitro load measurements to be examined can be compared with the Finite Element Analysis method.

Keywords: mandible, implant, finite element analysis, resection.

\section{Introduction}

In case of cancer and more closely oral cavity cancer in Hungary takes a very high number within the European Union. Considering the diagnosed oral cavity cancer, the male to female patient ratio is 5.3:1. The number of cancer patients from 1970 is constantly increasing. Nowadays 1700 patients die out of the 3000 diagnosed cases. Oral cavity cancers are however the easiest to detect. The doctors even in a regular dentist examination can detect and diagnose the cancer, in which case the illness can be cured with high success rate. This rate however radically decreases with time. In case of advanced state cancers the recovery is only possible with drastic measures 
and surgeries. The main factor of oral cavity cancer development is the inadequate oral hygiene, extent use of alcohol and tobacco products.

It is important to point out that cancers would cause metastasis in the bone as the third most common place after the lung and liver, in which case the jaw is also affected.

In case of mandible cancer resection is performed to remove it. During the surgery the affected area is determined by Computer Tomography and that specific part is than removed. The surgeon usually removes a larger area to ensure that there is no bone with cancer left. The complete resection method results in a loss of mechanical load carrying function and continuity of the mandible. Furthermore the self-picture of the patient is also affected, which can cause serious and overwhelming mental stress.

In the earlier times to gain more knowledge regarding the arising stresses in the bone and the connected implants, cadaver and bone ribbon examinations very conducted in laboratory settings. These examinations however are restricted by permissions, hence performing them is quite complicated. ${ }^{2,7}$ Because of this fact and the development of the technical science, the finite element analysis is becoming the more preferred solution. In the area of biomechanics the more complicated clinical cases become possible to be examined, this way supporting the creation of more optimal implants and the faster and more successful recovery of patients. ${ }^{3-5}$ In this thesis the different solutions of reconstruction in connection with the mandible resection is examined.

\section{Methods}

Implants are used in the reconstruction of resections. The implant manufacturers possess a large palette of implants that can be used for reconstruction. One of these is a regularly used so called reconstruction plate, which can be found in most of the manufacturers' catalogue with almost the same geometry. To fasten these plates commonly screws are used.

The mandible resections in clinical practice although not easily, but can be categorized into different groups. In reality the same case never occurs twice, however similarities can be found. In this current research the different sections where the cutting planes mostly occur are examined. 4 were highlighted and they were modeled with the help of the cutting planes (Figure 1).

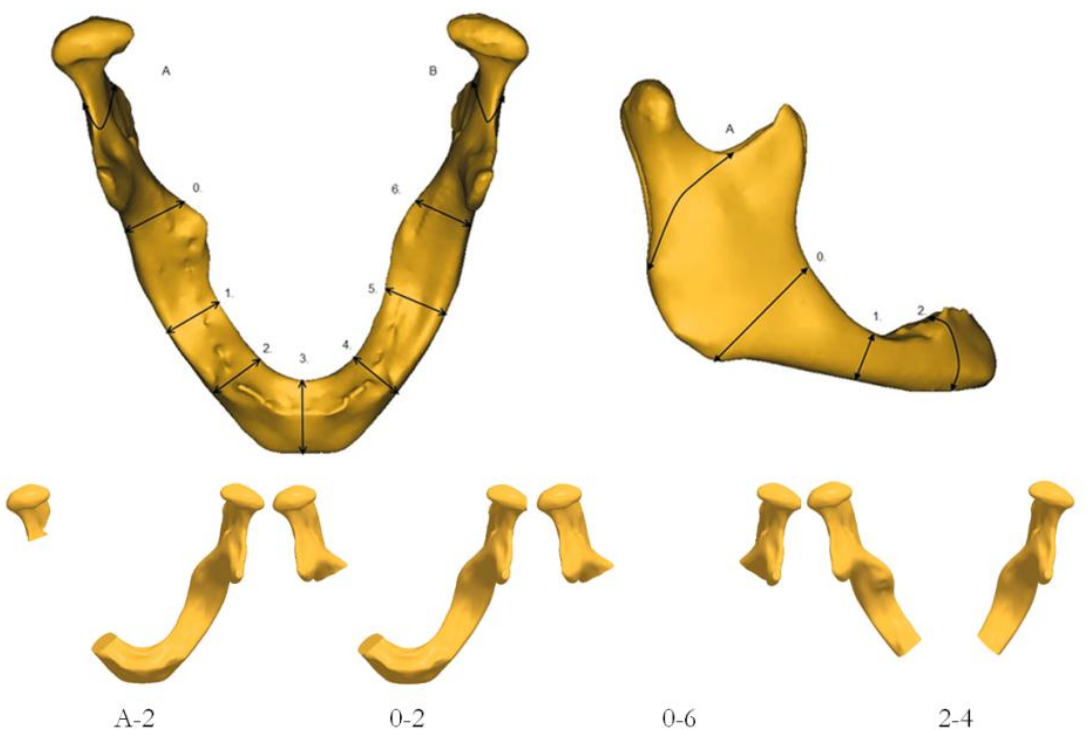

Figure 1. Different resections 
Regular reconstruction plates, which can be found at almost every manufacturer, were used with the prepared model in the mechanical reconstruction of the mandible. The plate thickness was set to be $3 \mathrm{~mm}$. The plate lengths used for the resection are always unique to conform to the surgery and to the length of the removed bone section. The plates during the examination were fixed with 3-3 bicortical screws on each resection side. The diameters were $2 \mathrm{~mm}$. The screws were examined with non-lock technology, which is secured by the traditional force-locking connection. In this current research the screws were not examined with the shape-locking lock technology. The material properties of the implants can be seen in the following table (Table 1). In case of the examined non-lock technique the pretention of the screws before the chewing load was also considered. Two different screw pretentions were examined in this study.

\begin{tabular}{|c|c|c|c|}
\hline \multicolumn{4}{|c|}{ MATERIALS } \\
\hline Name & Young modulus [GPa] & Ultimate Tensil Strenth [MPa] & Poisson \\
\hline $\begin{array}{c}\text { Plate - pure } \\
\text { Titanium }\end{array}$ & 102 & 680 & 0.3 \\
\hline $\begin{array}{c}\text { Screws - } \\
\text { Ti6Al7Nb }\end{array}$ & 105 & 900 & 0.33 \\
\hline
\end{tabular}

Table 1. Material properties

Realistic geometry was used during the construction of the jaw geometrical model. No simplification was applied. The model was created by the use of CBCT point cloud after the performed layer segmentation. The resection cuts on the mandible that were wished to be examined and the implant models to each case (reconstruction plates and the bicortical screws) were developed and positioned by CAD software (Figure 2).

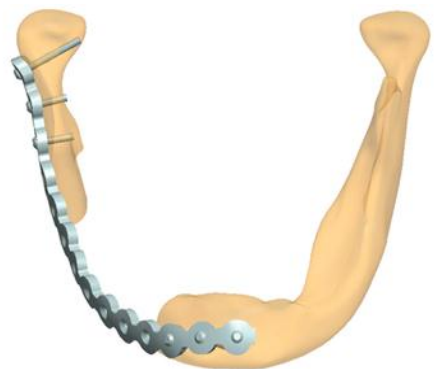

A-2

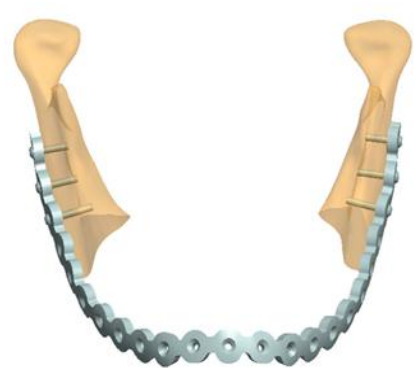

$0-6$

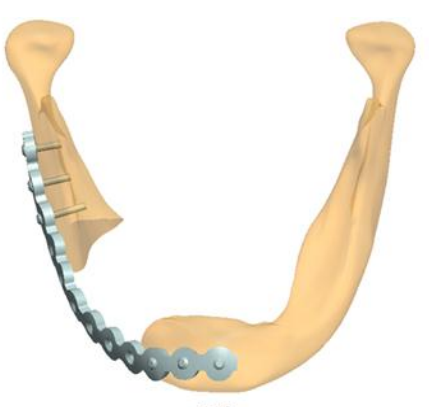

$0-2$

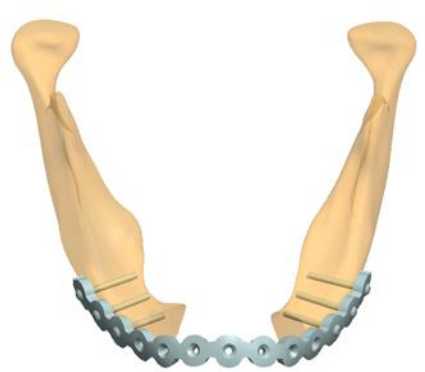

$2-4$

Figure 2. Different cases with implants

The necessary simulation mesh was created by using finite element software. During the mesh 10-node tetra elements were selected. Sub models were created in the area of the screws within the global model to support the refinement of the mesh and the examination of the different 
screw geometries. The mandible in the finite element model was applied with heterogeneous material properties to which the CBCT provided the necessary information. From the CBCT density results a mathematical formula was created to connect the material properties. Unique cylinders were made of selected materials and assembled into a plate for validation. Linear regression analysis was used to get correlation between $\mathrm{HU}$ value of the cylinders and relative density of the bone. The values of the Hounsfield Unit are known for the different commonly occurring materials in nature, for example distilled water (zero at standard temperature and pressure) and air (-1024 HU). The real material density was determined by the Hounsfield Unit (Equation 1).

$$
\text { density } \varrho=1.14264 \cdot \mathrm{HU}+309.4935\left[\mathrm{~g} / \mathrm{cm}^{3}\right]
$$

According to the written literature there is a connection between the linear elastic modulus and the density, hence the linear elastic modulus scale that reflects the bone structure can be covered (Equation 2). This change between 0-23000MPa.,

$$
E=0.024 \cdot \varrho^{1,777}[\mathrm{MPa}]
$$

The complete range was divided into 100 sections considering the material heterogeneous structure, where the material property can be changed by each tetra element. Next to the heterogeneous structure linearly elastic isotropic material properties were applied. The Poisson coefficient was set to 0.3 in case of the bone.

A cortical layer thickness was possible to be created with this model structure that is closer to reality. It was also very important to create a punctual model in the area of the screws which are used to fasten the reconstruction plates.

During the examination anatomically correct chewing muscle forces were used to describe the load on the mandible. These forces were used in earlier biomechanical researches. The three main applied muscle groups were the masseter, the temporalis and the medial pterygoid (Figure 3, Figure 4).

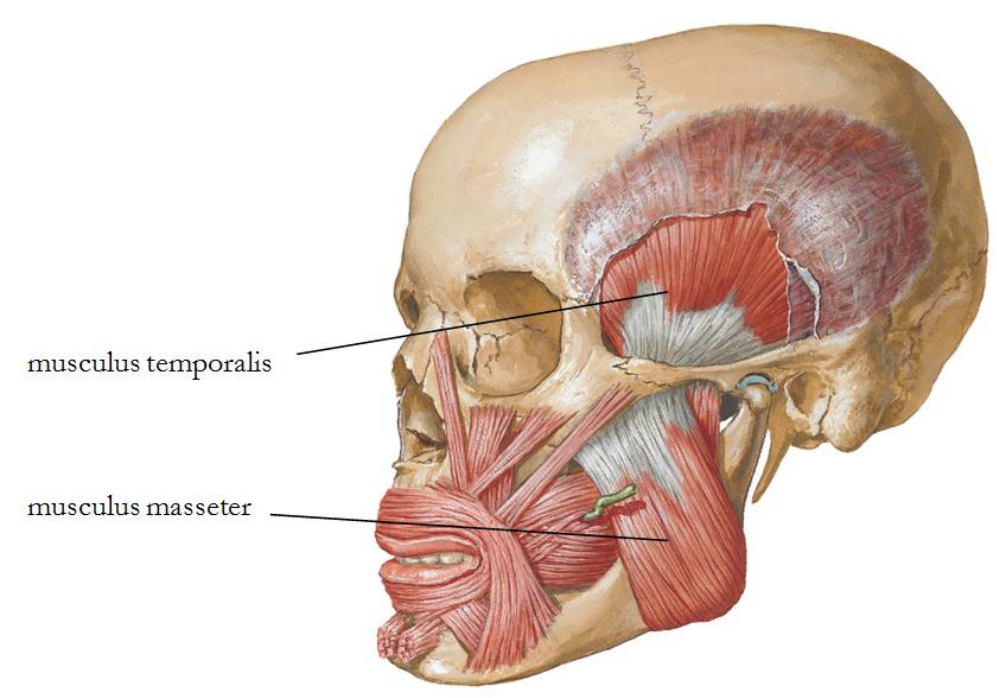

Figure 2. Temporalis and masseter muscles 


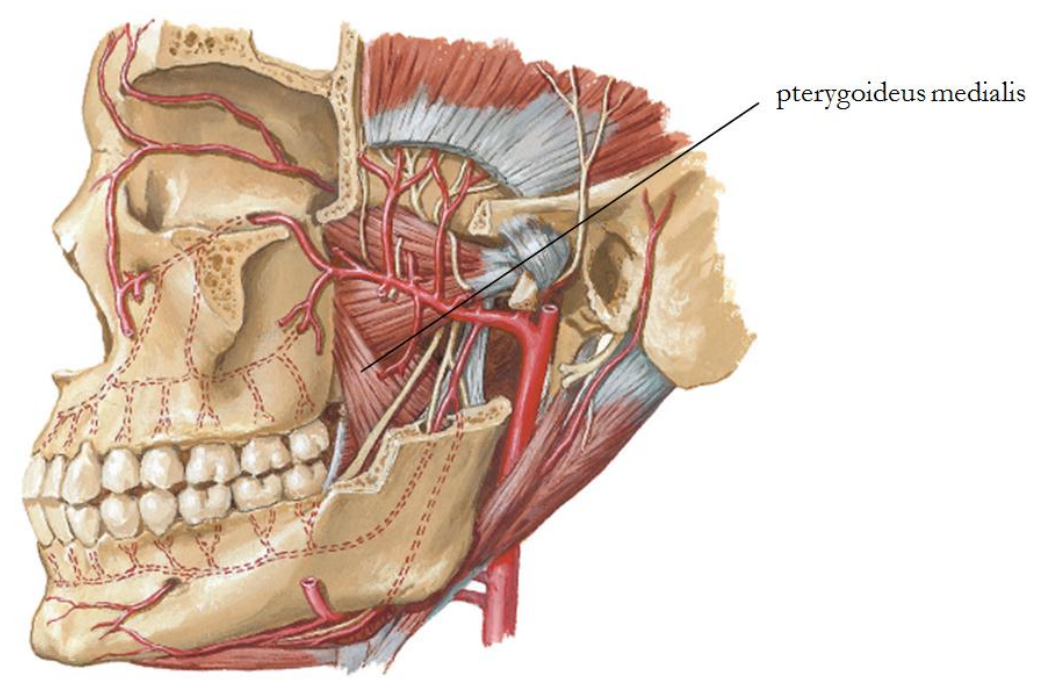

Figure 3. Medial pterygoid muscle

The loads and boundary conditions can be found on the following picture (Figure 5). The load cases in connection with each muscle group can be found in the Table 2 .

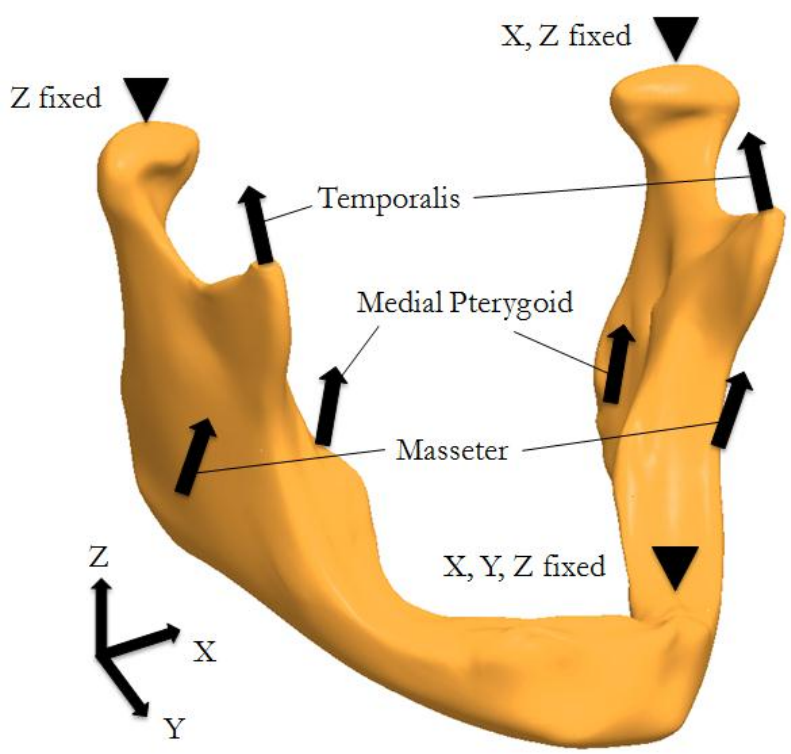

Figure 4. Boundary conditions and loads

\begin{tabular}{|c|c|c|c|}
\hline \multicolumn{4}{|c|}{ Loads in different directions [N] } \\
\hline & $\mathrm{X}$ & $\mathrm{Y}$ & $\mathrm{Z}$ \\
\hline Masseter & $50^{*}$ & 50 & 200 \\
\hline Medial Pterygoid & 0 & 50 & 100 \\
\hline Temporalis & 0 & -100 & 200 \\
\hline \multicolumn{2}{|c|}{${ }^{*}$ The X component is directed away from the mid-sagittal plane. }
\end{tabular}

Table 1. Muscle forces on mandible 
In every case between the bone and plates and between the screws and plates friction connections were defined because in the finite element analysis the non-lock cases were modeled. The coefficient is 0.3. [6] Shape-lock method was unable to use because of non-locking system. Force-locking connection was built with pretention force. One of the main focuses of the examination was the evaluation of the pretention force, therefore the finite element model was built up from two steps. In the first step the pretention of the plate is realized. Pretention was built by screw preloading. In the second step the chewing main load is applied. During the pretention of the plate in one case $50 \mathrm{~N}$, in the other case $100 \mathrm{~N}$ preload was defined for every screws in same time. The plate was pushed to the bone by these screws preloading. The pretention was provided this way. Bonded connection was defined between the bone and screws.

\section{Results}

During the evaluation of the results the clinical practice and the previous examination results were also considered. Based on this the bone area of screw number 3 and 4 were specifically alerting. These screws are located closest to the resection. In previous examinations the highest stress arose in these areas, which proves quite convincingly that the screw tear-out effect also occurs there. First step was to collect the stress data while using the pretention forces. In the second step the values while applying the main chewing load were gathered. The von Mises stress of the 0-2 and the 0-6 resection cases can be seen on the next figure (Figure 6).

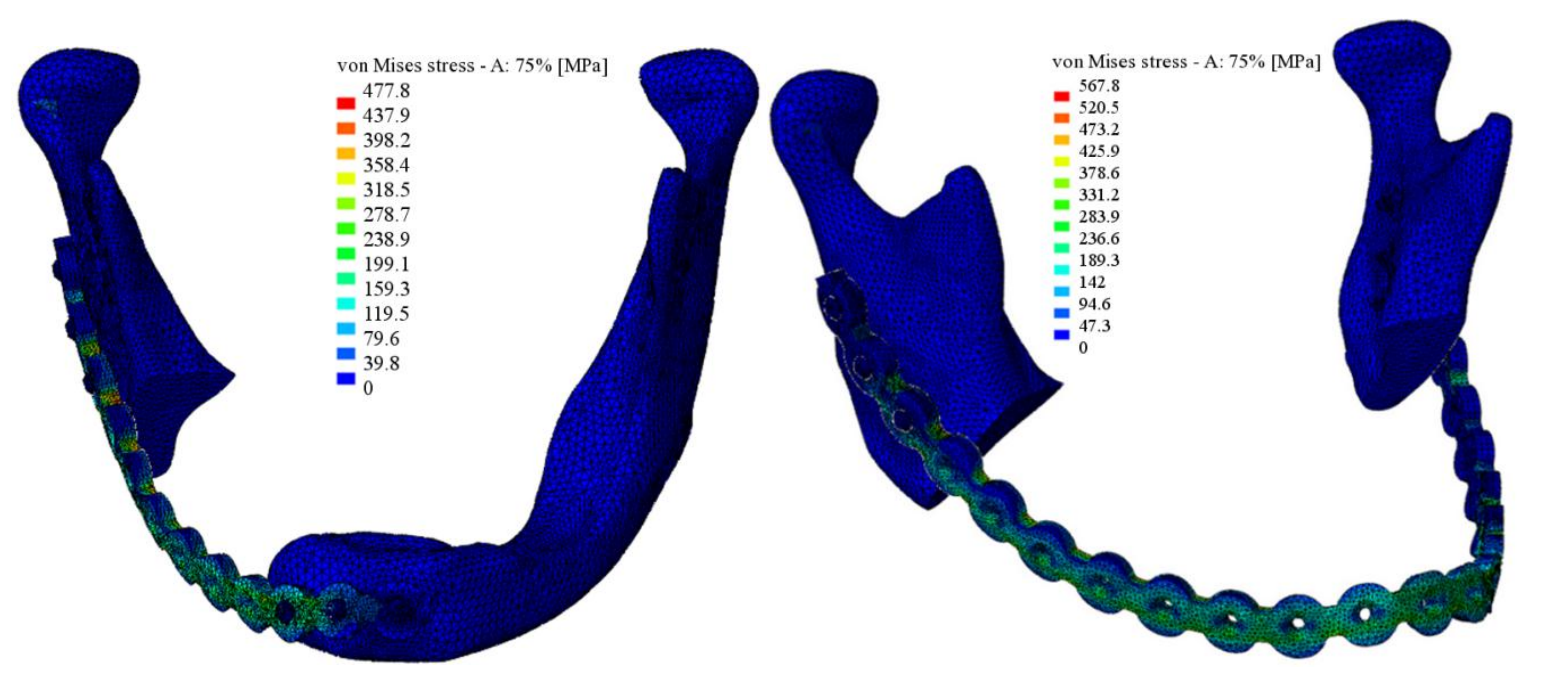

Figure 5. 0-2 and 0-6 resection cases, von Mises stress

The selected von Mises stresses were considered with nodal values on each element. The stresses are summarized in the following table (Table 3). 


\begin{tabular}{|c|c|c|c|c|c|c|c|c|c|}
\hline \multirow{2}{*}{$\begin{array}{c}\text { Maximal stress in } \\
\text { mandible at 3rd and } \\
\text { 4th screwplace }\end{array}$} & \multicolumn{3}{|c|}{ 50N PRELOAD } & \multicolumn{3}{c|}{ 100N PRELOAD } \\
\cline { 2 - 10 } & \multicolumn{2}{|c|}{ Step 1 } & \multicolumn{2}{|c|}{ Step 2 } & \multicolumn{2}{c|}{ Step 1 } & \multicolumn{2}{c|}{ Step 2 } \\
\cline { 2 - 10 } & Screw 3 & Screw 4 & Screw 3 & Screw 4 & Screw 3 & Screw 4 & Screw 3 & Screw 4 \\
\hline \multirow{4}{*}{ A-2 } & vM [MPa] & 18,3 & 49,1 & 66,7 & 300,1 & 25,6 & 58,9 & 60,5 & 246,2 \\
\cline { 2 - 10 } & S1 [MPa] & 23,7 & 55,8 & $-12,5$ & 344 & 32,1 & 67,2 & 73,9 & 281,4 \\
\cline { 2 - 10 } & $\mathrm{S} 3[\mathrm{MPa}]$ & 3,9 & 2,5 & $-84,38$ & 17,37 & 4 & 3,1 & 7,7 & 14,1 \\
\hline \multirow{4}{*}{$0-2$} & $\mathrm{vM}[\mathrm{MPa}]$ & 16,3 & 53,9 & 109,7 & 70,9 & 23,1 & 65,1 & 128,7 & 45,3 \\
\cline { 2 - 10 } & $\mathrm{S} 1[\mathrm{MPa}]$ & 19,6 & 60,6 & 126,9 & 80,4 & 27,7 & 73,6 & 148 & 49,4 \\
\cline { 2 - 10 } & $\mathrm{S} 3[\mathrm{MPa}]$ & 2 & 2 & 8,2 & 3,9 & 2,5 & 2,5 & 8,4 & 0,1 \\
\hline \multirow{4}{*}{$0-6$} & $\mathrm{vM}[\mathrm{MPa}]$ & 11,9 & 10,9 & 97,4 & 136 & 17,3 & 18,8 & 93,3 & 142,1 \\
\cline { 2 - 10 } & $\mathrm{S} 1[\mathrm{MPa}]$ & 13,6 & 15,3 & 112,9 & $-42,3$ & 19,8 & 26,6 & 108,1 & -44 \\
\cline { 2 - 10 } & $\mathrm{S} 3[\mathrm{MPa}]$ & 0,5 & 3,7 & 7,6 & $-118,4$ & 1 & 6,5 & 6,9 & $-196,6$ \\
\hline \multirow{3}{*}{$2-4$} & $\mathrm{vM}[\mathrm{MPa}]$ & 13,2 & 17,2 & 78,7 & 75,7 & 21,3 & 24,9 & 63,4 & 59,3 \\
\cline { 2 - 10 } & $\mathrm{S} 1[\mathrm{MPa}]$ & 15,2 & 19,5 & $-6,8$ & 86,6 & 23,6 & 27,7 & 71,3 & 67 \\
\cline { 2 - 9 } & $\mathrm{S} 3[\mathrm{MPa}]$ & 0,6 & 0,7 & $-90,7$ & 4,7 & 0,1 & 0,3 & 2,3 & 2,5 \\
\hline
\end{tabular}

Table 2. Stress values

The results are also shown on diagrams (Figure 7 and 8), where the stress difference in case of the use of $50 \mathrm{~N}$ and $100 \mathrm{~N}$ pretention force can be seen in each screw position case. It can be observed that in each case of screw number 3, for example A-2 and 0-6, the difference is minimal between the stresses while using the pretention force in the second chewing load. Considering screw number 3 in $0-2$ and 2-4 cases the previous statement does not apply as there is a real difference between the arising von Mises stresses. After the main load a significant difference can only be seen in the A-2 case. Further it can be stated that during the main chewing load between 0-2 and 2-4 cases there is no significant stress elevation compared to the pretention. In case of the chewing load the chance of implant tear-out is higher at screw number 3 in 0-2 case and at screw number 4 in A-2 case. This can be concluded from diagrams.

\section{Stress in mandible at 3rd screw place}

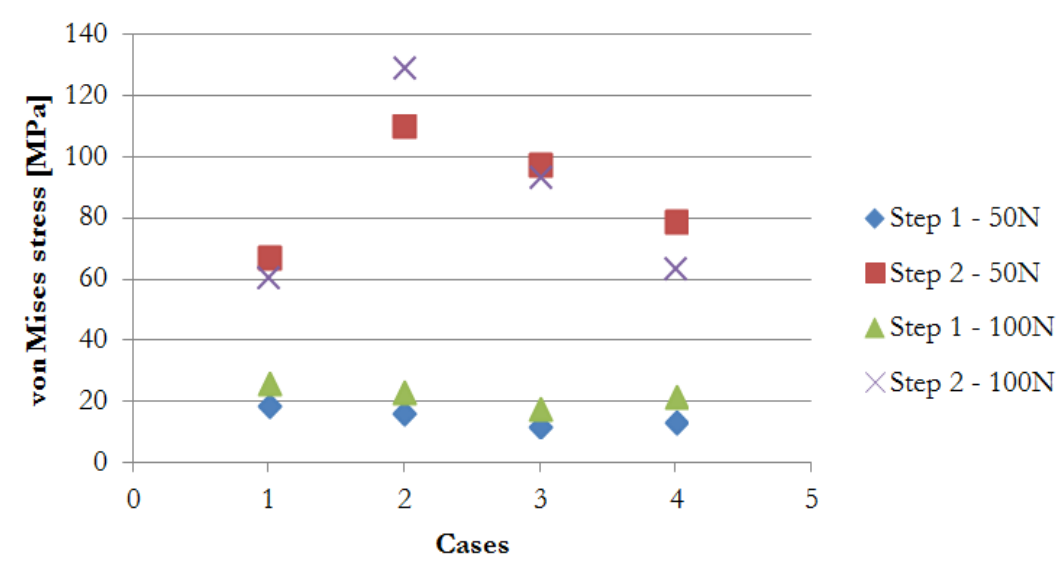

Figure 6. Different von Mises stress values at 3rd screw place 


\section{Stress in mandible at 4th screw place}

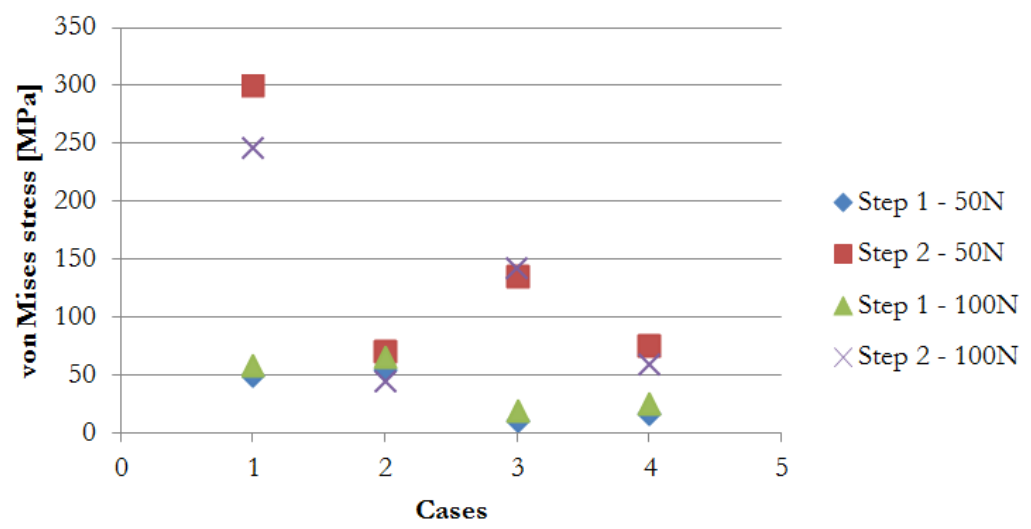

Figure 7. Different von Mises stress values at 4th screw place

The concluded finite element analysis gave the chance to estimate the stress in the areas of the screws in case of bicortical non- lock fastening technique during different resections. The results reflect which are the cases where the chance of screw tear-out is more likely to occur, furthermore in which cases the pretention forces have a higher effect in the arising stresses.

\section{REFERENCES}

1. Bujtar P, Avery C, Simonovics J, Sandor KG, Pan J. Refinements in osteotomy design to improve structural integrity: a finite element analysis study. The British Journal of Oral \& Maxillofacial Surgery 10/2012; DOI:10.1016/j.bjoms.2012.09.015

2. Avery CME, Best A, Patterson P, Rolton J, Ponter ARS. Biomechanical study of prophylactic internal fixation of the radial osteocutaneous donor site using the sheep tibia model. Brit J Oral Maxillofac Surg. 2007;45:441-6.

3. Simonovics J, Bodzay T, Váradi K. Study and examination of the implements used for securing pelvis bone. Biomechanica Hungarica III 2010;(1):215-23.

4. Bagi I. Finite element study of some parameters of bone fractures fixed with screws. Periodica Polytechnica - Mechanical Engineering 2011;55(1):57-61.

5. Lakatos É, Bojtár I. Stochastically Generated Finite Element Beam Model for Dental Research, Periodica Polytechnica Ser. Civ. Eng., 2009;53(1):3-8.

6. Shirazi Adl A, Dammak M, and Paiement G, Experimental determination of friction characteristics at the trabecular bone/porous coated metal interface in cementless implants. Journal of biomedical materials research, 1993;27(2):167-75.

7. Keller, T.S., Predicting the compressive mechanical behavior of bone. Journal of Biomechanics, 1994;27(9):1159-68.

I would like to thank Péter Mészáros for his help and support. 